\title{
The MSFC Collaborative Engineering Process for Preliminary Design and Concept Definition Studies
}

\author{
Jack Mulqueen $^{1}$ \\ Marshall Space Flight Center, MSFC, Alabama 35812 \\ David Jones ${ }^{2}$ \\ Marshall Space Flight Center, MSFC, Alabama 35812 \\ Randy Hopkins ${ }^{3}$ \\ SAIC/Marshall Space Flight Center, MSFC, Alabama 35812
}

\begin{abstract}
This paper describes a collaborative engineering process developed by the Marshall Space Flight Center's Advanced Concepts Office for performing rapid preliminary design and mission concept definition studies for potential future NASA missions. The process has been developed and demonstrated for a broad range of mission studies including human space exploration missions, space transportation system studies and in-space science missions. The paper will describe the design team structure and specialized analytical tools that have been developed to enable a unique rapid design process. The collaborative engineering process consists of integrated analysis approach for mission definition, vehicle definition and system engineering. The relevance of the collaborative process elements to the standard NASA NPR 7120.1 system engineering process will be demonstrated. The study definition process flow for each study discipline will be will be outlined beginning with the study planning process, followed by definition of ground rules and assumptions, definition of study trades, mission analysis and subsystem analyses leading to a standardized set of mission concept study products. The flexibility of the collaborative engineering design process to accommodate a wide range of study objectives from technology definition and requirements definition to preliminary design studies will be addressed. The paper will also describe the applicability of the collaborative engineering process to include an integrated systems analysis approach for evaluating the functional requirements of evolving system technologies and capabilities needed to meet the needs of future NASA programs.
\end{abstract}

\section{MSFC Advanced Concepts Office Team Structure}

The Marshall Space Flight Center (MSFC) Advanced Concepts Office (ACO) utilizes a unique collaborative engineering approach for conducting mission architecture and concept definition studies in support of pre-phase A and phase A project formulation of potential future NASA missions. The Advanced Concepts office uses a permanently co-located team of specialized multi-discipline design engineers and a streamlined systems-based design process to perform a broad range of technical studies. The MSFC Advanced Concepts Office design team conducts Earth-to-orbit launch vehicle systems studies, robotic and science mission studies and human space exploration studies. The multi-discipline design team provides a unique capability to perform integrated systems analysis, mission analysis, system architecture assessments and subsystem design analysis for concept definition studies. The team structure of the MSFC Advanced Concepts Office is shown in Figure 1. Each team is composed of design analysts representing the design disciplines listed for each team.

\footnotetext{
${ }^{1}$ Aerospace Engineer, MSFC Advanced Concepts Office/ED04

${ }^{2}$ Aerospace Engineer, MSFC Advanced Concepts Office/ED04

${ }^{3}$ Aerospace Engineer, MSFC Advanced Concepts Office/ED04
} 


\section{Advanced Concepts Office}

\section{Advanced Concepts Office Management}

\section{Earth-to-Orbit Transportation Team:}

\begin{tabular}{l}
\hline Systems Analysis \\
\hline Systems Engineering \\
\hline Mission \& Trajectory Analysis \\
\hline Configuration \& Design \\
\hline Structures \\
\hline Propulsion \\
\hline Launch Vehicle Weights \& Sizing
\end{tabular}

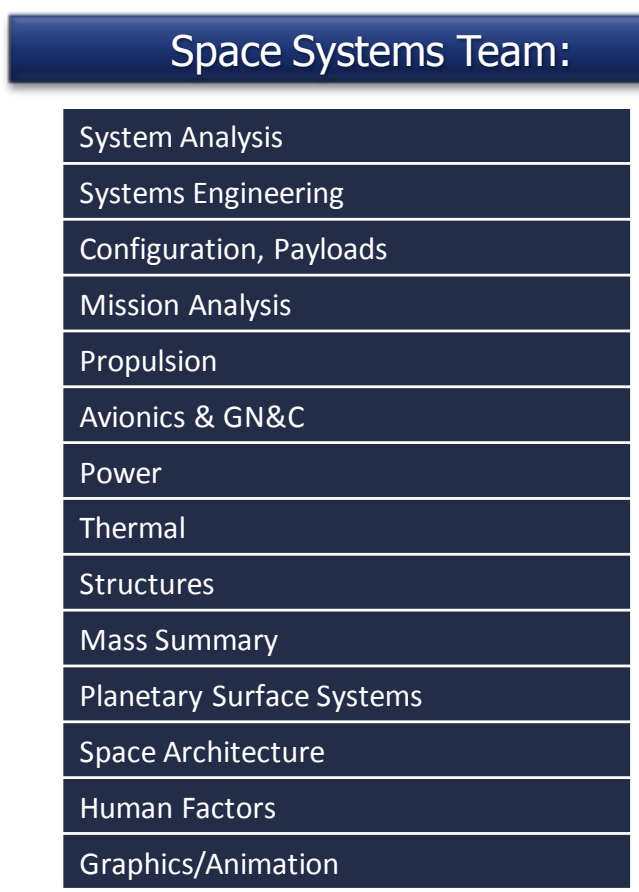

Figure 1. Advanced Concepts Office Team Structure

\section{Collaborative Engineering Tools}

The Advanced Concepts Office uses a wide array of computer design analysis tools. The use of industry standard computer tools allows high fidelity engineering analysis based on widely accepted modeling techniques and capabilities. In many studies it is possible to incorporate higher fidelity analyses such as finite element thermal and structural modeling into conceptual pre-phase studies. This capability allows consideration of subsystem trades and design analysis that would otherwise be beyond the scope of pre-phase A conceptual studies. Due to the specialized nature of many Advanced Concepts studies, many additional in-house developed tools have been created to perform spacecraft performance analysis and subsystem design analysis. These specialized tools allow efficient analysis for preliminary design trades and concept definition. The Earth-to-orbit analysis tools developed by the Advanced Concepts Office allow extremely efficient analysis of launch vehicle design options, including multiple liquid and solid propulsion systems, stage configurations and payload carrying options. Also among the unique capabilities possessed by the Advanced Concepts office is the ability to perform broad parametric sizing and performance analysis of space transportation system architectures. The capability to perform integrated analysis of Earth-to-orbit launch vehicle and in-space transportation architectures provides a unique optimization capability. The mission analysis tools and capabilities to perform Earth-to-orbit and in-space trajectory analysis and orbital analysis provide a significant capability that is integral to the comprehensive analysis of mission architectures. The primary engineering analysis and design tools used by the Advanced Concepts Office are summarized in Table 1. 
Table 1. Advanced Concepts Office Design Tools

\begin{tabular}{|l|l|}
\hline \multicolumn{1}{|c|}{ Design Tool } & \multicolumn{1}{c|}{ Function } \\
\hline POST - Program for Optimizing Space Trajectories & Launch vehicle trajectory and performance analysis \\
\hline LVA - Launch Vehicle Analysis & Launch vehicle structural analysis \\
\hline INTROS - Integrated Rocket Sizing Program & Launch vehicle sizing and performance analysis \\
\hline Copernicus, MIDAS, MaNE, & In-Space trajectory analysis \\
\hline Thermal Desktop and in-house thermal analysis tools & Spacecraft thermal analysis \\
\hline FEMAP w/NX NASTRAN, Hypersizer & Spacecraft structural analysis \\
\hline In-house power system tools & Solar array sizing and spacecraft power system analysis \\
\hline In-house attitude control system tool & GN\&C subsystem sizing \\
\hline In-house propulsion system tool & Propulsion system analysis \\
\hline COPA - Computerized Optimum Performance Analysis & In-space stage sizing and performance analysis \\
\hline Transportation Architecture Analysis & Parametric transportation system analysis \\
\hline ProEngineer & Vehicle design and configuration \\
\hline 3D Studio & Animation development \\
\hline vDOT & Design process and data management \\
\hline
\end{tabular}

\section{Collaborative Systems Based Design Process}

The collaborative design process used by the Advanced Concepts Office has evolved over many years of conducting a wide range of conceptual design studies in support of virtually every NASA initiative and program over the past several decades. The process has been modified to adapt to changes in NASA emphasis from technology development to science missions to major flight system programs. The collaborative design process is based on a comprehensive systems based approach that is valid across the broad spectrum of NASA programs and conceptual analysis. The foundation of the collaborative design process is sequential systems analysis process that begins with mission and system functional analyses to identify the top level mission objectives and requirements. The design process consists of three iterative processes for mission definition, vehicle definition and system engineering. The Advanced Concepts Office collaborative design process is illustrated in Figure 2. The mission definition process includes analysis tasks to define the mission profile and identify the range of mission options that comprise the mission trade space. Based on the mission requirements and mission trade space, initial spacecraft vehicle options and design ground rules and assumptions are defined. This process is often iterative in nature, as mission requirements are derived from analysis of the mission and vehicle trade space. The results of the mission definition iterations contribute to the definition of the mission concept.

The vehicle definition process is closely linked to the mission definition process. The vehicle definition is based on requirements derived from the mission analysis which becomes the basis for vehicle performance and subsystem design assumptions. The initial vehicle design ground rules and assumptions not only shape the vehicle options and subsystem trades, but may eventually serve as the initial vehicle and subsystem requirements. The subsequent analysis of subsystem design trades and subsystem design analysis contribute to the definition of the vehicle concept.

In parallel with the mission and vehicle definition processes, system engineering processes are conducted to the extent possible for pre-phase A and conceptual design studies. These initial system engineering activities are compliant with the to the established NASA project formulation guidelines as specified in NASA Procedural Requirements document NPR 7120.1. The system engineering products developed at this early stage of program maturity often contribute to the specified system engineering products required for the Mission Concept Review (MCR) program key decision point (KDP) as listed in Table 2. The initial system engineering activities consist of initial concept of operations and requirements definition. In addition initial system risk identification is often a valuable component of the system analysis. The system engineering analyses, integrated with the mission and vehicle concepts contribute to the overall definition of the system concept. 


\section{Collaborative Design Process}

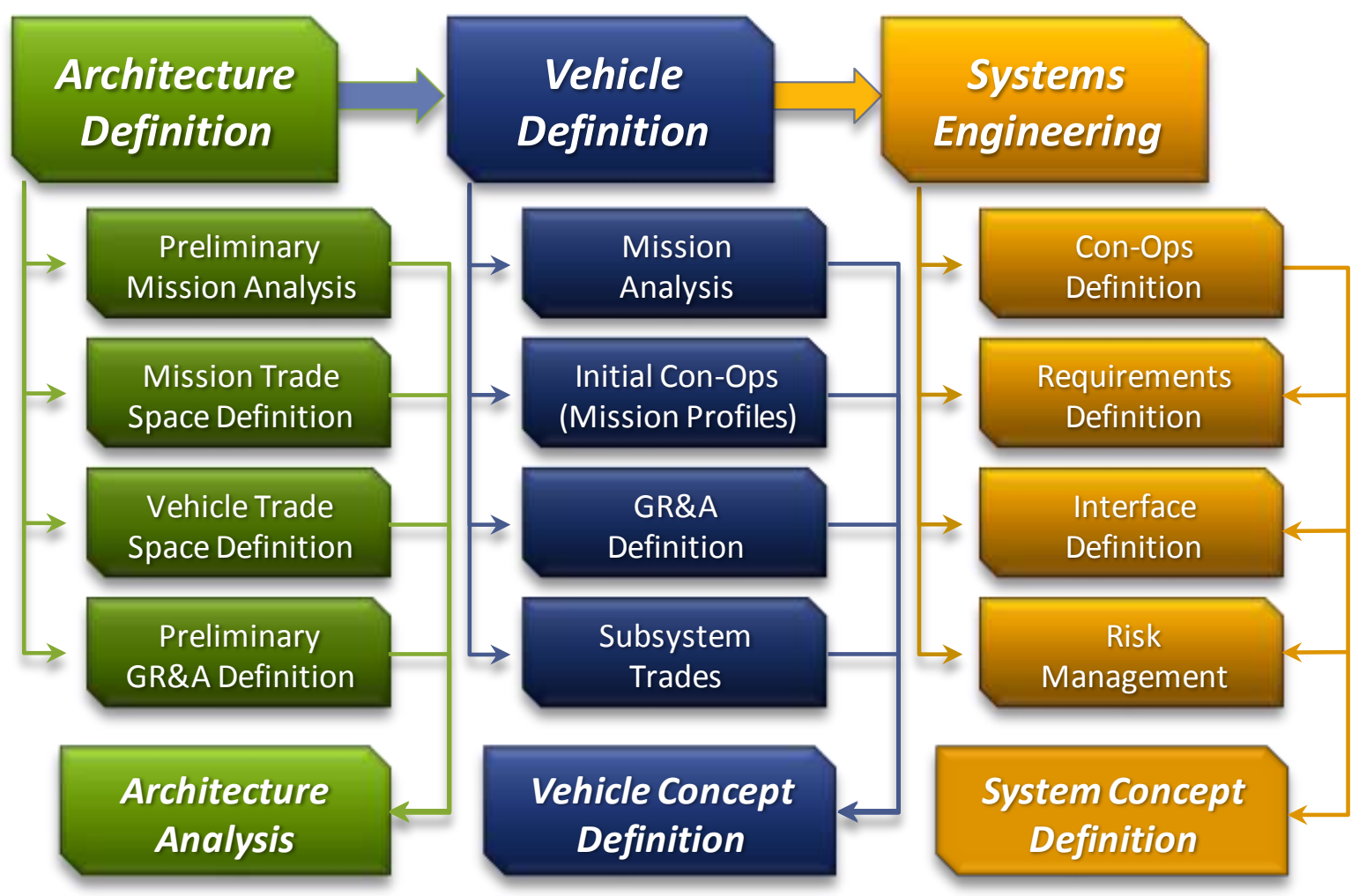

Figure 2. Systems Based Collaborative Design Process

Table 2. Systems Based Collaborative Design Process

\begin{tabular}{|l|l|}
\hline \multicolumn{1}{|c|}{ MCR Criteria (NPR 7123.1A) } & \multicolumn{1}{c|}{ Pre-Phase A Study Products } \\
\hline Mission Goals and Objectives & Mission Concept Definition \\
\hline Preliminary Requirements & $\begin{array}{l}\text { Mission Requirements Inputs } \\
\text { Spacecraft Requirements Inputs }\end{array}$ \\
\hline Evaluation of Alternative Concepts & $\begin{array}{l}\text { Trade Study Results } \\
\text { Alternative Configuration Evaluation }\end{array}$ \\
\hline Concept of Operations & $\begin{array}{l}\text { Mission Analysis Results } \\
\text { Design Reference Mission Summary }\end{array}$ \\
\hline De-Scope Options & De-Scope Options Summary \\
\hline Risk Assessment and Mitigation Strategies & Project Risk Analysis Inputs \\
\hline Technical Plans for Next Phase & Draft Phase A Technical Plan Inputs \\
\hline Measures of Effectiveness & Mission and Vehicle trade studies \\
\hline Life Cycle Support Strategies & Project Cost and Schedule Inputs \\
\hline Technically Feasible Mission Concept & Mission Analysis Results \\
& Preliminary Mission Architecture \\
\hline Credible Cost \& Schedule Estimates & Cost and Schedule Inputs \\
\hline Evaluation of Existing Assets & Trade Study Results \\
& Preliminary Mission Architecture \\
\hline
\end{tabular}

American Institute of Aeronautics and Astronautics 


\section{IV. mDot Design Process}

The process used by the Advanced Concepts Office is based on an iterative process which includes all mission and vehicle design disciplines working in a coordinated effort to converge to the desired analysis result or design solution. In most cases the iterations are based on reaching a converged system mass estimate. For this reason, the design process is called the mDot Design Process. Figure 3 shows the basic steps and information flow of the mDot process. The key to the efficiency of this process is the collaboration between all of the design disciplines. The process is based on iterative analysis and transmission of analysis results to all related design disciplines so dependencies and interactions between subsystems can be addressed within each design iteration. Figure 3 shows a simplified representation of the design process. Many of the interactions and feedback loops between design disciplines are not shown. Basically, the design process begins after initial study planning is completed. The study planning phase consists of gathering information from the study customer and defining the mission concept. The initial mission and vehicle trade space is also defined during the study planning process. Once the planning is complete, the design process is initiated with the definition of design ground rules and assumptions. Usually a joint design session is held in which each design discipline defines initial ground rules and assumptions. The initial ground rules and assumptions are documented and serve as the starting point for the design iteration. The ground rules and assumptions are revisited each iteration because in many cases they must be adjusted as the study progresses. The mission analysis tasks are closely related to the ground rules and assumptions since the mission profile, trajectory, orbit analysis and mission delta-velocity budget are all crucial to beginning many of the subsystem analyses. In many cases, an initial vehicle configuration is defined as a starting point for the subsystem design trades. Once there is sufficient information available to begin the vehicle subsystem analysis, the actual design iterations can begin. Within each design iteration data is passed between subsystem designers in a highly interactive fashion. Efficient communication, data management and documentation of design results are crucial to maintain the efficiency of the design process. In addition, each discipline maintains a keen awareness of the interactions and dependencies between subsystems. This allows overall system optimization of design results.

\section{Mdot Design Process}

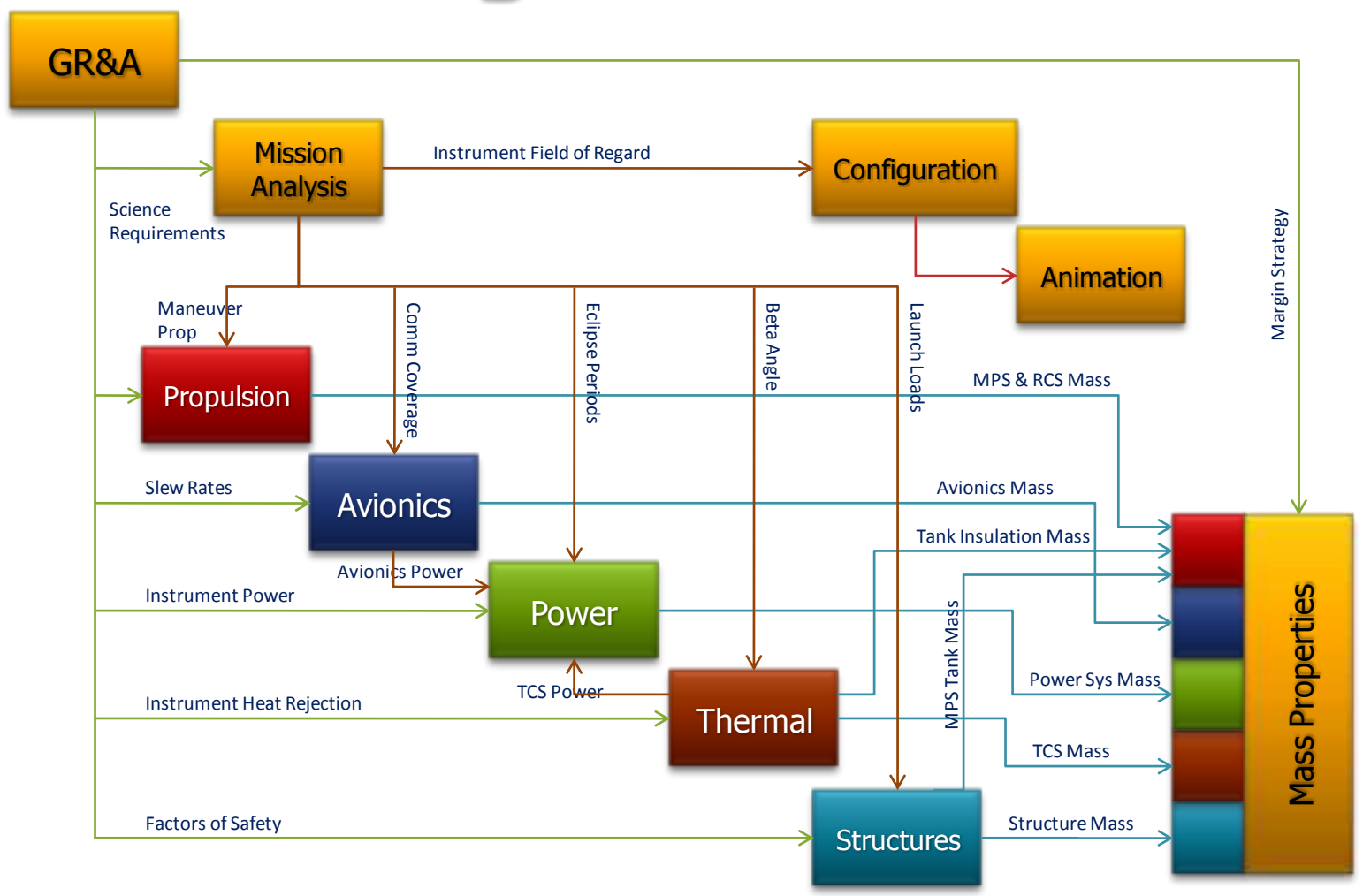

Figure 3. mDot Collaborative Design Process

American Institute of Aeronautics and Astronautics 
The objective of the design iteration process is to converge on the design concept for each subsystem. Mission analysis is performed each iteration to evaluate the vehicle performance, including propulsion system propellant requirements. As the design iterations progress, the convergence of the design solution is usually measured by the change in vehicle dry mass and propellant mass. When the iterations are complete the study results are documented. In many studies additional system engineering activities augment the vehicle concept definition. As stated earlier, the system engineering tasks may include requirements analysis, risk identification or development of other system engineering products. The Advanced Concepts has developed a standard study documentation process to compile the study results and present them to customers and stakeholders.

\section{Integrated Systems Analysis}

The collaborative design process can be applied to a wide range of study types and different levels of systems analysis. While the collaborative design processes are well suited for vehicle concept definition studies, the same process provides a framework for evaluating system interactions and dependencies at a much higher level as well. The systems based approach has been used to define and evaluate space transportation architectures, evaluate mission infrastructure options and assess technology needs. The systems analysis approach is illustrated in Figure 4. The approach represents a sequential process for defining and evaluating future NASA programs and mission options. The process begins with broad mission and system functional assessments. Based on the mission requirements, the mission architecture options and elements can be defined. The architecture assessment usually consists of trade studies and evaluation of mission options, spacecraft transportation options and definition of mission payloads. Assessment of future NASA programs often also includes evaluation of emerging technology capabilities and development needs. The integrated system based approach and collaborative engineering process developed by the MSFC Advanced Concepts Office provides a unique capability for evaluating evolving NASA program and missions in a highly effective and efficient manner.

\section{Systems Analysis Applications}

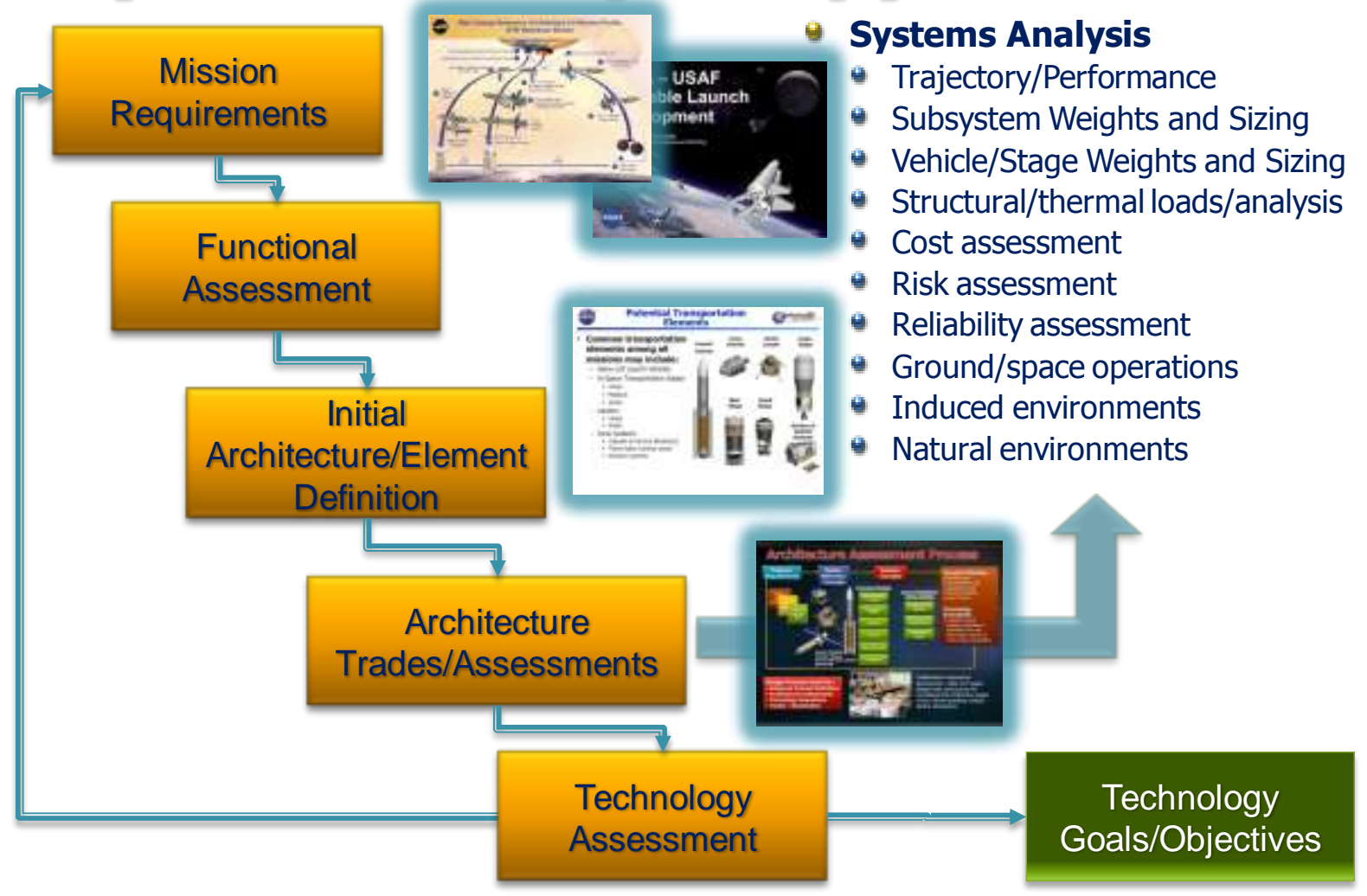

Figure 4. Systems Based Analysis Process 